\title{
A REAÇÃo DE CICLIZAÇÃo DE PRINS: UMA ESTRATÉGIA EFICIENTE PARA SÍNTESE ESTEREOSSELETIVA DE ANÉIS TETRAIDROPIRÂNICOS SUBSTITUÍDOS
}

\author{
Mário Luiz A. A. Vasconcellos* \\ Departamento de Química, Universidade Federal da Paraíba, Campus I, 58059-900 João Pessoa - PB, Brasil \\ Leandro S. M. Miranda \\ Núcleo de Pesquisas de Produtos Naturais, Universidade Federal do Rio de Janeiro, Bloco H, Centro de Ciências da Saúde, \\ Ilha do Fundão, 21941-590 Rio de Janeiro - RJ, Brasil
}

Recebido em 5/5/05; aceito em 27/9/05; publicado na web em 12/4/06

\begin{abstract}
THE PRINS CYCLIZATION REACTION: AN EFFICIENT STRACTEGY FOR THE STEREOSELECTIVE SYNTHESIS OF SUBSTITUTED TETRAHYDROPYRAN RINGS. The Prins cyclization reaction has significantly advanced in the last years as demonstrated by a number of applications described in the literature. The objective of this report is to introduce this powerful synthetic methodology to the undergraduate and graduated student, since it is rarely presented in an organic synthesis formal course. This reaction is, in some cases, the methodology of choice for the preparation of natural products or drugs that present the tetrahydropyrane moiety in their structures. In this report we show some aspects of this reaction, including mechanism, scope and limitations.
\end{abstract}

Keywords: Prins cyclization; tetrahydropyrans; stereoselective synthesis.

\section{INTRODUÇÃOO}

A síntese estereosseletiva de produtos naturais é uma das vertentes da Química que vem apresentando um crescimento contínuo e exponencial nas últimas décadas. Este destacado interesse tem origem principalmente na enorme diversidade estrutural e na variada atividade das biomoléculas, sendo normalmente uma fonte inspiradora infindável para desenvolvimento de novos fármacos. Entre esta inesgotável variedade estrutural, podemos destacar a importância dos anéis do tipo tetraidropirano substituídos, presentes em inúmeros produtos naturais ${ }^{1-3}$. Podemos encontrá-los, por ex., em carboidratos como a glicose $\mathbf{1}$, nos anéis A e B do antibiótico Monensina $\mathbf{2}^{4}$, no anel A do antibiótico 17-deoxiroflamicoína $3^{5}$, nos anéis A, B, C, D, E, F e G da neurotoxina Brevetoxina B $4^{6}$ e nos anéis A, B, C e D dos anti-tumorais naturais Forboxazola 5a e $5 b^{7}$ (Figura 1).

Diversas estratégias seletivas vêm sendo desenvolvidas objetivando a preparação de tetraidropiranos substituídos, podendo-se citar como exemplos, as reações de hetero-Diels-Alder ${ }^{8-10}$, reações de Michael intramoleculares ${ }^{11-13}$, ciclização de dióis e de $\delta$-hidroxicetonas $^{4,14,15}$, reação de iodolactonização ${ }^{16-18}$, selenoeterificação de álcoois insaturados ${ }^{19}$, abertura de epóxidos ${ }^{20-22}$ e a reação de ciclização de Prins ${ }^{23-37}$.

O objetivo deste trabalho é a apresentação das principais potencialidades e limites da reação de ciclização de Prins, pouco apresentada nos cursos formais de síntese orgânica oferecidos na graduação e pós-graduação, mas que vêm se destacando nos últimos anos como uma metodologia poderosa de escolha para preparação de vários produtos naturais que apresentam, em seu arcabouço, tetraidropiranos substituídos.

\section{Definições}

A reação de Prins (que é a precursora da moderna reação de ciclização de Prins) é muito antiga, datando de $1919^{38}$, e apresenta até o momento mais de 700 artigos publicados na literatura. A reação de

*e-mail: mlaav@quimica.ufpb.br

$$
\underbrace{R^{2}}_{R^{1}}
$$

$$
\begin{aligned}
& \text { Anel Tetraidropirana substituído } \\
& \mathrm{R}=\mathrm{H} \text {, alquila, arila, heteroátomos }
\end{aligned}
$$
la, heteroátomos

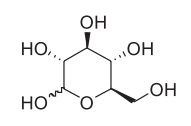

$\alpha$-Glicopiranose

1

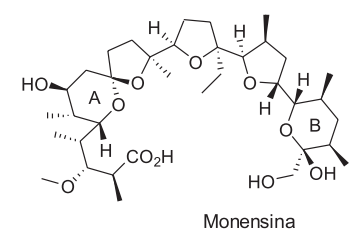

2
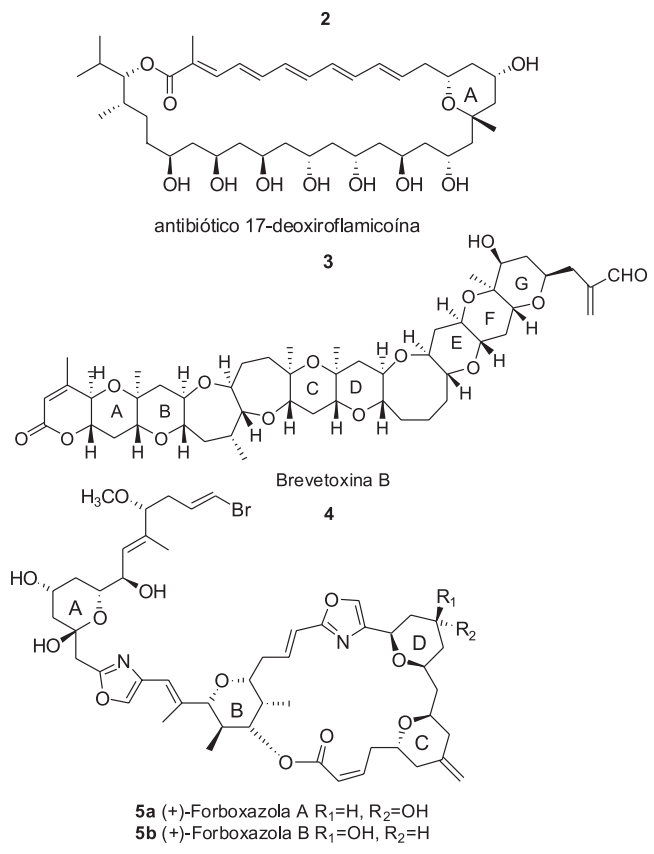

Figura 1. Tetraidropiranos substituídos como fragmento estrutural de Produtos Naturais 
Prins consiste, em sua essência, na condensação entre olefinas com formaldeído, feita em meio aquoso e catalisada por ácidos minerais (Esquema 1). Os produtos, em geral obtidos nesta condensação, são formados como misturas complexas de 1,3-dioxanos 13, 1,3-glicóis 9, derivados tetraidropirânicos $\mathbf{1 2}$ e álcoois insaturados alílicos $\mathbf{1 0}$ e homoalílicos $\mathbf{8}$, sendo a composição da mistura dependente das condições experimentais empregadas (Esquema 1).

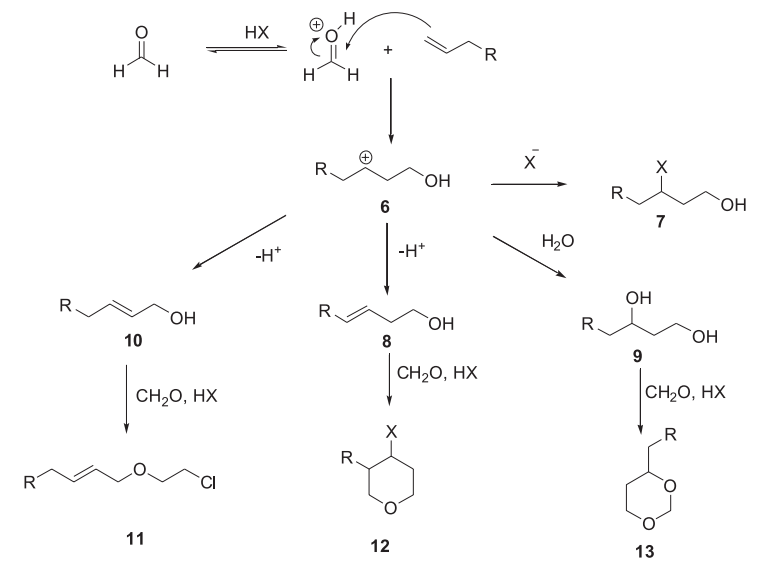

Esquema 1. A reação de Prins

No Esquema 1, podemos notar que a presença de água no meio reacional conduz o intermediário $\mathbf{6}$ à formação de 1,3-glicóis $\mathbf{9}$, assim como de 1,3-dioxanas 13. A formação do derivado alquil-4halo-tetraidropirano 12 é obtida por intermédio do álcool homoalílico 8 .

Devido à formação de uma mistura complexa de produtos, a reação de Prins permaneceu muitos anos sem aplicações sintéticas muito relevantes. Entretanto, a partir da publicação, em 1955, por Hanschke e Gendorf ${ }^{39}$, do primeiro exemplo em que houve a reação direta entre álcool homoalílico puro 14 e formaldeído, catalisada por ácido sulfúrico em aquecimento, levando ao tetraidropirano 15 como produto principal em bom rendimento (Esquema 2), houve uma revitalização na expectativa sintética deste tipo de reação.

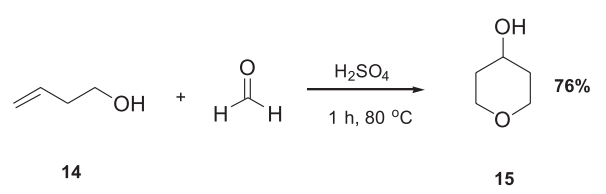

Esquema 2. Primeiro exemplo da reação, que seria futuramente denominada de reação de ciclização de Prins

A partir desse artigo e de um posterior descrito por Stapp ${ }^{40}$, entre outros ${ }^{24}$, este tipo particular de reação de Prins começou a ser chamada de reação de ciclização de Prins. Esta reação vem, desde então, despertando um grande interesse sintético na comunidade científica. Até o momento, mais de 100 artigos foram descritos na literatura (consultando somente as palavras Prins e "cyclization" como palavras-chave cruzadas, no "Chemical Abstract (C.A.) on line", existiam 125 referências até o momento da submissão deste artigo), sendo que este crescimento vem ocorrendo de forma exponencial.

A explosão no interesse sobre esta reação, principalmente a partir do final da década passada, está diretamente relacionada a sua versatilidade sintética, ao fato desta apresentar bons rendimentos químicos, como também nas altas diastereosseletividades cis/ trans obtidas entre os substituintes do anel tetraidropirânico. Este tipo particular de reação de Prins é o enfoque deste artigo. Desta maneira, definimos a ciclização de Prins ${ }^{23-37}$ como o acoplamento entre álcoois homoalílicos (ou éteres) e aldeídos (ou acetais), mediada principalmente por um ácido de Lewis ou de Brönsted $\left(\mathrm{AlCl}_{3}\right.$, $\mathrm{AlBr}_{4}, \mathrm{SnCl}_{4}, \mathrm{SnBr}_{4}, \mathrm{BF}_{3} . \mathrm{Et}_{2} \mathrm{O}, \mathrm{TMSBr}, \mathrm{InCl}_{3}, \mathrm{H}_{2} \mathrm{SO}_{4}$, entre outros) com mostrado no Esquema 3.

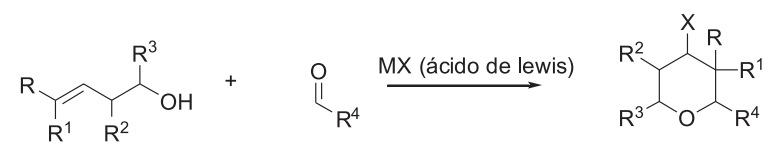

Esquema 3. A reação de ciclização de Prins: $R, R^{l}, R^{2}, R^{3}, R^{4}$ e $R^{5}=H$, alquila ou arila; $X=O H$, Halogênios, $H$

\section{ASPECTOS MECANÍSTICOS \\ Tetraidropirano versus tetraidrofurano e a diastereosseletividade cis versus trans}

Uma das vantagens da reação de ciclização de Prins é o fato desta conduzir exclusivamente a tetraidropiranos, quando se utilizam como substratos álcoois homoalílicos terminais $\left(\mathrm{R}, \mathrm{R}^{1}=\mathrm{H}\right)$, ou álcoois homoalílicos de geometria $E\left(\mathrm{R}^{1}=\mathrm{H}\right.$ e R diferente de $\mathrm{H}$, Esquema 3). Esta regiosseletividade pode ser racionalizada admitindose o mecanismo aceito para esta reação ${ }^{41}$, mostrado no Esquema 4.

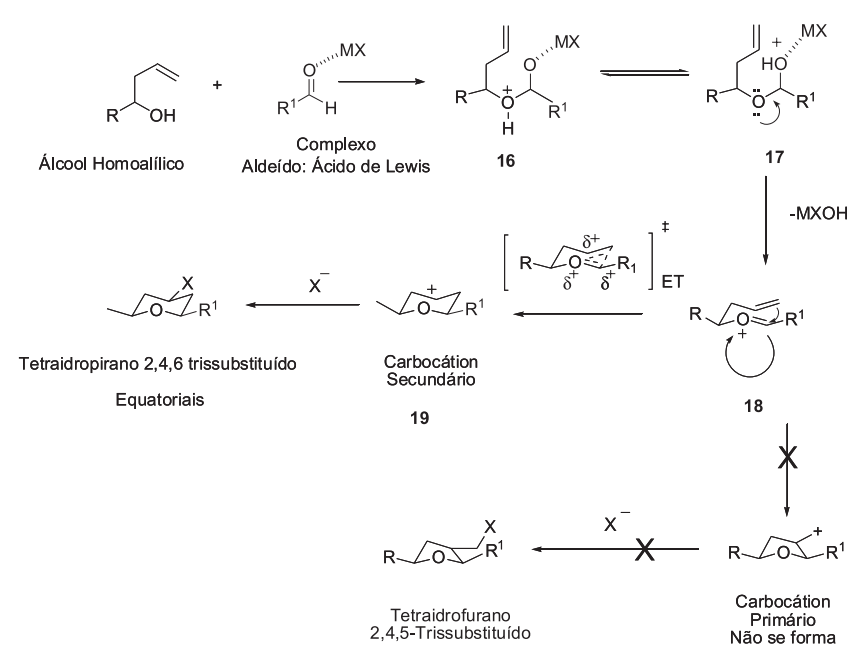

Esquema 4. Mecanismo aceito para a reação de ciclização de Prins

Segundo este mecanismo proposto, há a formação do acetal intermediário 16 a partir da reação nucleofílica entre os elétrons $n$ da hidroxila do álcool homoalílico e o carbono eletrofílico do aldeído ativado pelo ácido de Lewis. Este acetal gera, então, o íon oxônio 18, o qual, na etapa de ciclização, assume uma conformação do tipo cadeira, de modo que os substituintes assumam preferencialmente uma posição pseudo-equatorial no estado de transição (o que é mais estável), conduzindo ao carbocátion secundário 19. A subseqüente captura do carbocátion 19 pelo haleto $\mathrm{X}^{-}$(proveniente do ácido de Lewis) ocorre, dependendo das condições reacionais utilizadas, preferencialmente na posição equatorial. A partir deste mecanismo, podese entender o porquê de álcoois homoalílicos terminais conduzirem exclusivamente à formação do anel tetraidropirânico. Note neste Esquema mecanístico que, para que houvesse a possibilidade da formação de um anel tetraidrofurânico (de 5 membros), a reação deve- 
ria passar pela formação de um carbocátion primário (o que é energeticamente muito desfavorável) no lugar do secundário 19. Entretanto, é conhecido que quando álcoois homoalílicos de geometria $Z$ são usados como substratos na reação de ciclização de Prins, há a possibilidade da formação concorrente de anéis de cinco membros. Neste caso, a geometria da ligação dupla irá definir a posição deste substituinte no estado de transição, como pode ser compreendido observando o Esquema 5.

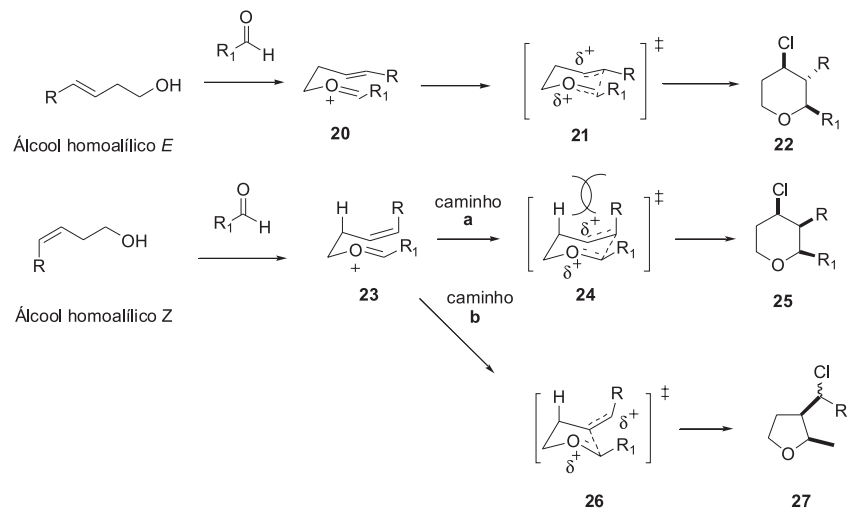

Esquema 5. Regiosseletividade entre a formação de anéis de 6 e 5 membros, na ciclização de Prins, em função da geometria E ou Z do álcool homoalílico

Note que, no caso da reação com álcoois homoalílicos de geometria $E$, o estado de transição apresenta o substituinte $\mathrm{R}$ da ligação dupla na posição pseudo-equatorial (o que é relativamente mais estável) como pode ser observado em 21, conduzindo ao anel tetraidropirânico 2,3,4-trissubstituído 22 com o substituinte na posição $\mathrm{C}_{3}$ de geometria relativa trans em relação aos das posições 2 e 4 (substituintes nos $\mathrm{C}_{2}, \mathrm{C}_{3}$ e $\mathrm{C}_{4}$ em posições tri-equatoriais). Por outro lado, caso a reação venha a ocorrer com um álcool homoalílico com a ligação dupla de geometria relativa $Z$, dois "caminhos mecanísticos" competitivos podem ser aventados (caminhos a e b, Esquema 5). No primeiro (caminho a), o estado de transição $\mathbf{2 4}$ apresentaria o substituinte $\mathrm{R}$ na posição pseudo-axial, conduzindo ao anel tetraidropirânico 25. Entretanto, este posicionamento pseudo-axial do substituinte $\mathrm{R}$ da ligação dupla gera uma interação estérea desfavorável 1,3-diaxial entre $\mathrm{R}$ e $\mathrm{H}$ em 24. Note que este tipo de interação desfavorável não existe no estado de transição 26, caminho b, para a ciclização em anel de 5 membros (tetraidrofurânico), acarretando assim a concorrência entre anéis de 5 e de 6 membros.

Uma outra potencialidade desta reação que deve ser destacada é a alta diastereosseletividade na formação de anéis tetraidropirânicos funcionalizados nas posições 2,4 e 6 , onde normalmente os substituintes encontram-se na configuração relativa cis, (tri-equatoriais), na maioria dos experimentos relatados. Vejamos a seguir, como este fato foi recentemente interpretado.

\section{A diastereosseletividade segundo o modelo de Alder $^{42}$}

Em um precioso estudo teórico computacional, usando a teoria do Funcional da Densidade (B3LYP/6-31G*), Alder e colaboradores concluíram que o favorecimento da seletividade 2,4,6-cis, na reação de ciclização de Prins pode ter origem na geometria do intermediário catiônico envolvido na reação de ciclização de Prins. De acordo com a interpretação de Alder, a interação entre um par de elétrons $n$ do oxigênio com mais dois pares de elétrons $\sigma$ (um em cada uma das ligações $\sigma$ C-C do anel cíclico) e o orbital $\mathrm{p}$ do carbocátion torna este sistema catiônico 28 particularmente estável (Figura 2). Esta estabilidade foi interpretada baseada no caráter aromático deste intermediário. Para que esta "aromaticidade" possa ocorrer, é necessário que o $\mathrm{H}$ do carbono em $\mathrm{C}_{4}$ ocupe obrigatoriamente a posição pseudo-axial, tornando todos os 6 elétrons conjugados do sistema cíclico ( $n$ e $\sigma$ ) co-planares, estando desta forma de acordo com a Regra de Hückel. Esta conformação particular favorece o ataque nucleofílico em 28 (Figura 2) pela face exo conduzindo, conseqüentemente, ao produto 2,4,6-tri-equatorial.

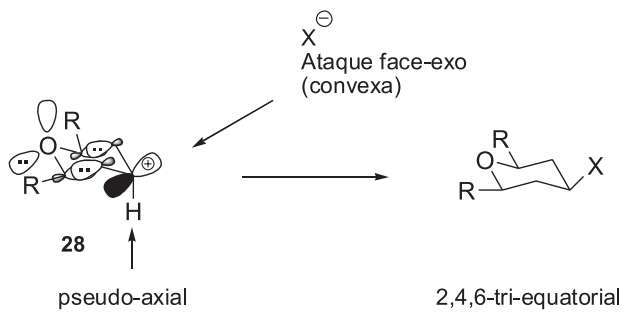

Figura 2. Modelo de Alder para a estereosseletividade 2,4,6-tri-equatorial, na reação de ciclização de Prins

\section{A seletividade Axial em $\mathrm{C}_{4}$ : o modelo de Rychnovsky ${ }^{43,44}$}

Contrapondo-se à teoria proposta por Alder, Rychnovsky e colaboradores propuseram que o caráter aromático do intermediário catiônico per si, formado durante a reação de ciclização de Prins, não explica totalmente a estereosseletividade desta reação. Rychnovsky descreveu um protocolo experimental para obtenção estereosseletiva de haletos $(\mathrm{Br}, \mathrm{I})$ na posição axial em $\mathrm{C}_{4}$ do anel tetraidropirânico. Neste relato, Rychnovsky utilizou $\alpha$-acetoxi-ésteres 29 como precursores do íon oxônio intermediário, além de TMSBr , AcBr ou TMSI em diclorometano e lutidina, levando ao produto de ciclização de Prins em altos rendimentos e em altas estereosseletividades axiais em $\mathrm{C}_{4}$ (Esquema 6). Por outro lado, o uso de $\mathrm{SnBr}_{4}$, nas mesmas condições reacionais, conduz seletivamente ao produto equatorial. Uma possível explicação para este fato foi apresentada por Rychnovsky ${ }^{43}$ e está sumarizada no Esquema 6.

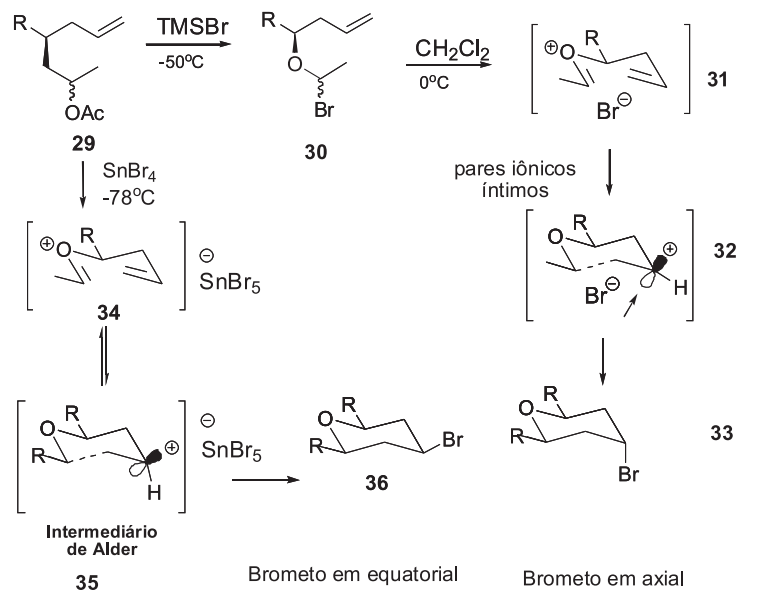

Esquema 6. Modelo de Rychnovsky

Podemos notar nesta proposta mecanística que quando o brometo de trimetilssilano (TMSBr) é a fonte de íon brometo, há a formação dos pares iônicos íntimos 31 e 32, que reagem imediatamente, favorecendo o ataque do íon brometo sobre o carbocátion de maneira intra-par-iônico, sendo este ataque na posição endo, conduzindo ao produto axial 33. Por outro lado, quando o ácido de 
Lewis é o $\mathrm{SnBr}_{4}$, há a formação do íon 34, onde o pentabrometo de estanho $\left(\mathrm{SnBr}_{5}^{-}\right)$é o contra-íon. De acordo com Rychnovsky, a menor nucleofilicidade do íon $\mathrm{SnBr}_{5}$ - versus o $\mathrm{Br}^{-}$"livre" (oriunda do brometo de trimetilssilano no caso anterior) permite a formação do par-iônico-separado por solvente 35, favorecendo o ataque pela face exo (convexa), conduzindo à formação do brometo equatorial 36.

\section{O rearranjo de oxônia-Cope: uma reação colateral ${ }^{44-46}$}

Devido à presença do intermediário oxônio $\mathbf{1 8}$, a reação de ciclização de Prins pode apresentar, como uma reação colateral, o rearranjo de oxônia-Cope (Esquema 7). Em um estudo recente ${ }^{44}$, Rychnovsky descreve vários estudos que comprovam que o rearranjo de oxônia-Cope ocorre preferencialmente quando um oxônio pode se transformar em um outro oxônio, sendo este mais estável que o inicial ou com energias muito parecidas ${ }^{44}$.

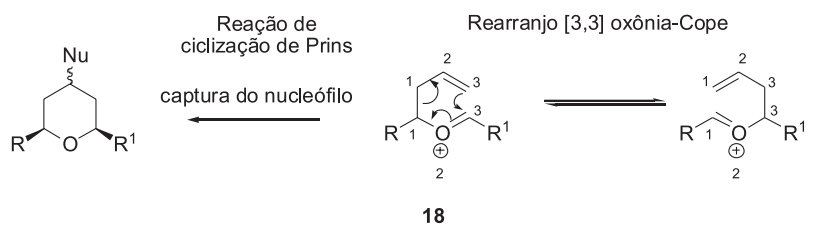

Esquema 7. Rearranjo [3,3] oxônia-Cope

Destacamos a seguir, um exemplo ilustrativo da ocorrência evidente deste rearranjo, observado pelo nosso grupo de pesquisas $^{35}$. Neste estudo, o produto principal da reação de ciclização de Prins entre o (+/-)-1-fenil-1-hidroxi-but-3-eno e o propanal foi obtido em $85 \%$ de rendimento químico. Entretanto, foram detectados em 7 e $8 \%$ de rendimento, respectivamente, os produtos (+/-)- cis 37 e (+/-)-cis 38. A ocorrência de um rearranjo de oxônia-Cope é a única forma de justificar a observação destes tipos de subprodutos simétricos $^{35}$ (Esquema 8).

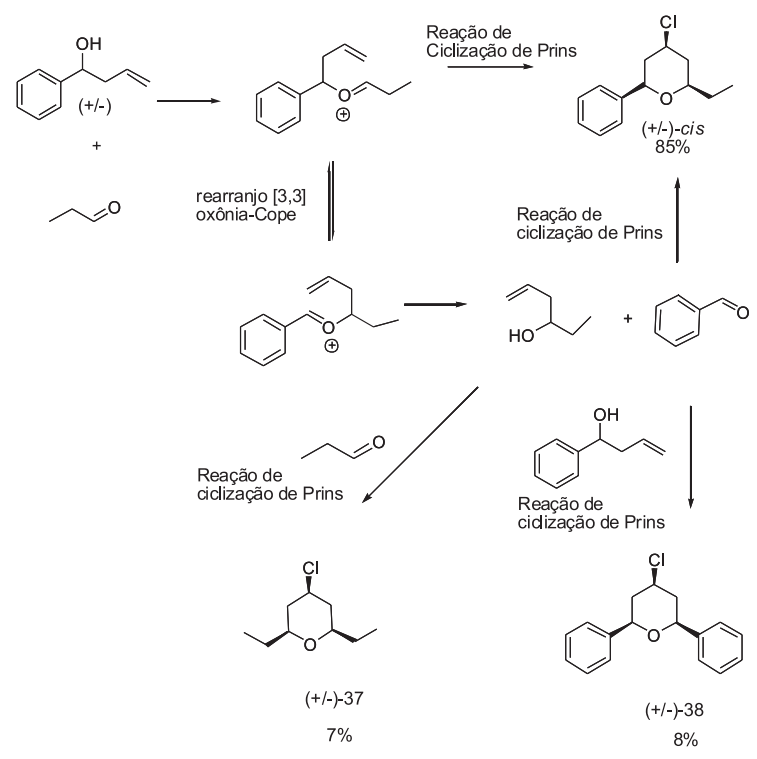

Esquema 8 . Subprodutos oriundos do rearranjo de oxônia-Cope

Em geral, o rearranjo de oxônia-Cope não acarreta maiores problemas na reação de ciclização de Prins. Entretanto, perda de pureza ótica de álcoois homoalílicos precursores, durante o curso reacional, já foi relatada ${ }^{45-47}$. Este pode vir a ser um fator limitante para a reação de ciclização de Prins, caso haja necessidade do controle da configuração absoluta do produto final. Por ex., a utilização de álcoois homoalílicos substituídos por grupos aromáticos "ricos em elétrons" (grupos aromáticos ligados a substituintes elétron-doadores), conduz ao produto oriundo de oxônia-Cope em grande extensão, devido à estabilidade adicional do íon oxônio formado durante o curso reacional ${ }^{45,46}$. Entretanto, algumas soluções para estas limitações vêm sendo apresentadas, como veremos mais adiante em sínteses enantiosseletivas, como a da (-)-centrolobina ${ }^{47}$.

\section{EXEMPLOS ILUSTRATIVOS DE PREPARAÇÃO DE PRODUTOS NATURAIS OU FÁRMACOS VIA REAÇÃO DE CICLIZAÇÃO DE PRINS}

No final da década de 1980, na década de 90 e principalmente neste início deste milênio, vêm surgindo na literatura vários exemplos onde a reação de ciclização de Prins mostrou-se muito eficiente como etapa-chave na construção do esqueleto tetraidropirânico, existente em Produtos Naturais ou não Naturais. Nesta seção, passaremos a mostrar alguns destes exemplos ilustrativos, objetivando salientar as potencialidades desta reação (Figura 3).
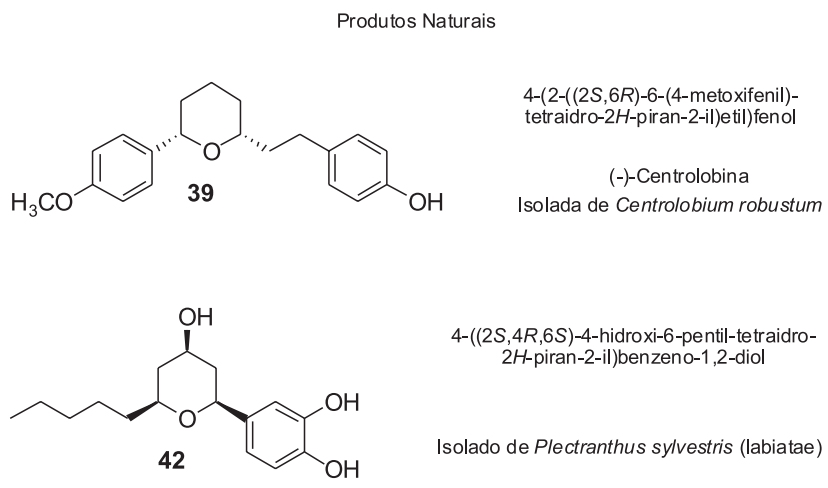
$2 H$-piran-2-il)benzeno-1,2-diol

Isolado de Plectranthus sylvestris (labiatae)

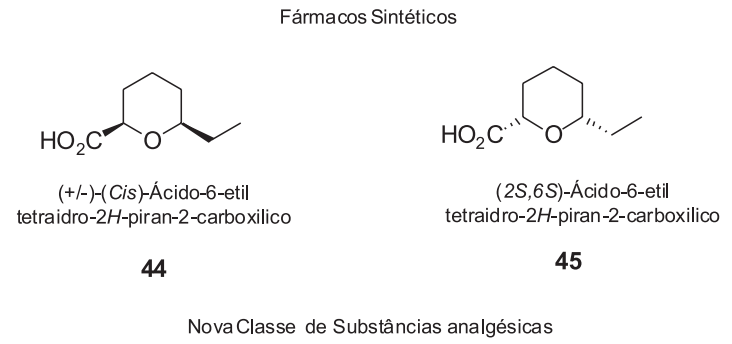

Figura 3. Algumas substâncias que foram sintetizadas usando a reação de ciclização de Prins como etapa-chave na formação de tetraidropiranos substituídos

A (-)-centrolobina 39 é um antibiótico isolado das cascas de Centrolobium robustus e teve sua estrutura elucidada em $1964^{48,49}$. Uma síntese de 39, que venha a usar a reação de ciclização de Prins como etapa-chave na construção do anel tetraidropirânico, deverá considerar a presença deste grupo aromático rico em elétrons $\left(\mathrm{PhOCH}_{3}\right)$ ligado diretamente ao sistema tetraidropirânico, que representa um perigo à pureza ótica do álcool homoalílico. Isto ocorre, como já comentamos, devido à estabilidade adicional do íon oxônio gerado no mecanismo reacional, facilitando a reação colateral de oxônia-Cope. Entretanto, uma solução elegante para este problema foi descrita por Richnovsky ${ }^{47}$, onde o grupo $\mathrm{OCH}_{3}$ foi substituído pelo grupo OTs ( $p$-toluenossulfonato, um grupo elétron-atrator, Esquema 9, 40 e 41). Esta modificação de 
substituinte no anel aromático minimizou a reação de oxônia-Cope, conduzindo à preparação enantiosseletiva da (-)-centrolobina 39, em ótimo rendimento químico e pureza ótica.

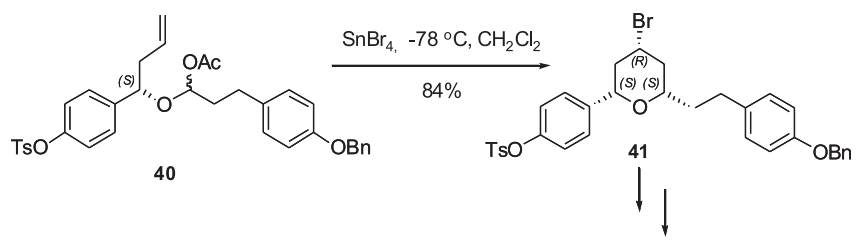

(-)-centrolobina 39

Esquema 9. Reação de ciclização de Prins como etapa-chave na síntese enantiosseletiva da (-)-centrolobina 39

A síntese enantiosseletiva do catecol $\mathbf{4 2}$, isolado de Plectrantus sylvestris (Figura 3), é também um belo exemplo das potencialidades da reação de ciclização de Prins ${ }^{46}$. Como no caso anterior, os grupos elétron-doadores (grupos $\mathrm{OH}$ catecólicos) favorecem a reação colateral de oxônia-Cope, conduzindo a produtos colaterais e à racemização. Entretanto, quando estes grupos hidroxila foram acetilados, levando ao álcool homoalílico 43 , como estratégia para evitar epimerização, houve minimização deste problema. Os grupos acetatos foram posteriormente convertidos ao grupo catecol de 42, como mostrado no Esquema 10.

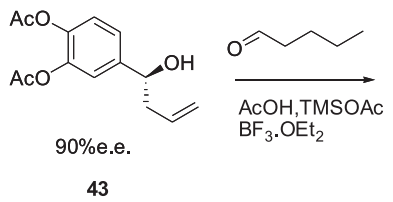

43

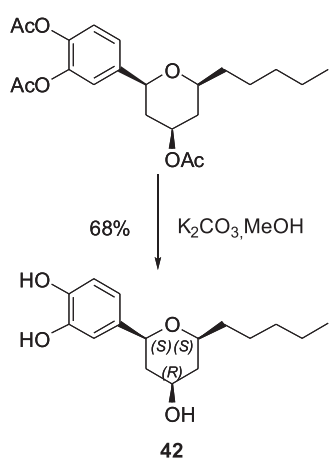

$80 \%$ e.e.

\section{Esquema 10. Síntese do catecol natural 42}

O ácido carboxílico 44 (Figura 3) que foi inicialmente apontado como o princípio ativo de uma planta medicinal brasileira do gênero Vitex, mostrou-se como um forte analgésico não esteroidal, quando comparado à dipirona, e atua bem mais rapidamente que a morfina ${ }^{36}$. Recentemente esta substância foi preparadas pelo nosso grupo de pesquisas, de maneira diastereosseletiva ${ }^{36}$, originando (+/-)-44, e enantiosseletiva, originando (-)-45 $\mathbf{5}^{37}$, usando como metodologia sintética a reação de ciclização de Prins nas etapaschave das sínteses. Por ex., a síntese enantiosseletiva de $\mathbf{4 4}$ foi feita mediante a reação de ciclização de Prins entre álcool homoalílico oticamente enriquecido 46 (e.e. 87\%) com propanal. Neste exemplo, não houve observação de nenhum produto co-lateral, bem como houve a manutenção total da pureza ótica, indicando que a reação de oxônia-Cope foi evitada. Desta forma, o ácido (-)-45 foi preparado com total manutenção da estereoquímica original (Esquema 11).

\section{EXPANSÃO DAS POTENCIALIDADES DA REAÇÃO DE CICLIZAÇÃO DE PRINS}

Outros exemplos vêm aparecendo na literatura, ampliando cons-

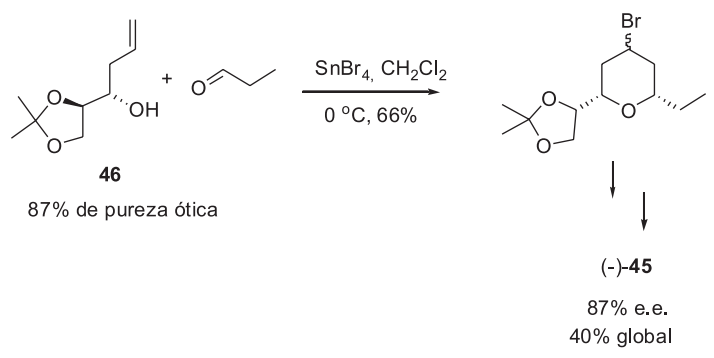

Esquema 11. Sintese do analgésico não esteroidal 42

tantemente a potencialidade desta reação. Podemos destacar a reação de Aza-Prins ${ }^{50,51}$, Tio-Prins ${ }^{52}$ e a ciclização de Prins que conduz à formação de anéis de mais de seis membros ${ }^{53}$, como mostrado na Figura 4.

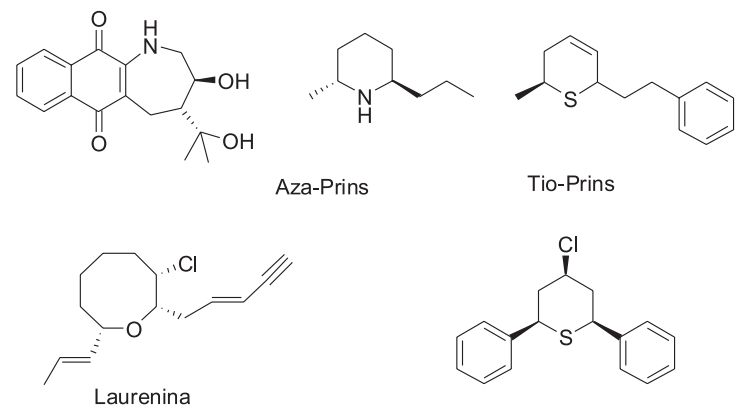

Figura 4. Exemplos de expansões das potencialidades da reação de ciclização de Prins

A reação de ciclização de Prins é também usada em reações em sequiência ("tandem”), em protocolos de aldol-Prins ${ }^{54,55}$, bem como as de Prins-pinacol ${ }^{56-59}$. Como exemplo ilustrativo destas reações seqüenciais ("tandem”), mostramos no Esquema 12, uma reação onde o dieno (-)-47, preparado na forma enantiomericamente pura via catálise assimétrica ${ }^{60}$, reage com o 4-fenilbutanal, mediante uma reação aldólica seguida de ciclização de Prins ${ }^{55}$, conduzindo ao produto tetraidropirânico $\mathbf{4 8}$, em $72 \%$ de rendimento químico. Neste exemplo, a geometria do carbono $\mathrm{C}_{6}$ é totalmente controlada pela geometria do carbono assimétrico do dieno 47. No carbono $\mathrm{C}_{4}$, houve um controle parcial da estereoquímica (84\% e.d, produto preferencialmente equatorial) e no carbono carbinólico, não houve controle da estereosseletividade.

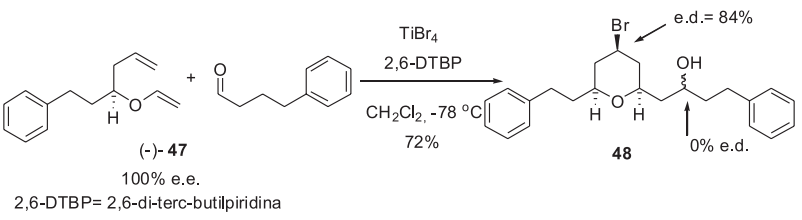

Esquema 12. Titânio (IV) promovendo reação seqüencial aldol-ciclização de Prins

\section{CONCLUSÃO E PERSPECTIVAS}

A reação de ciclização de Prins é um método atual e eficiente para preparação de produtos naturais (ou não naturais) que contenham, na sua estrutura, o anel teraidropirânico substituído. A estereoquímica relativa formada durante o curso reacional, entre as posições nos carbonos $\mathrm{C}_{2}$ e $\mathrm{C}_{6}$ do anel tetraidropirânico, é prefe- 
rencialmente de configuração cis. No $\mathrm{C}_{4}$, o controle da geometria depende do tipo de ácido de Lewis escolhido. Assim, podemos escolher a metodologia que envolve a formação de par-iônico-íntimo, conduzindo preferencialmente à configuração relativa trans entre $\mathrm{C}_{4}$ com $\mathrm{C}_{2}$ e $\mathrm{C}_{6}$ ou a metodologia que envolve a formação do par-iônico separado por solvente, que conduz preferencialmente à geometria relativa cis entre $\mathrm{C}_{4} \operatorname{com} \mathrm{C}_{2}$ e $\mathrm{C}_{6}$. Acreditamos que as maiores evoluções nesta área deverão estar concentradas no desenvolvimento de novas metodologias para controle da estereoquímica em todas as posições do anel tetraidropirânico. O uso de outros tipos de heterociclos formados (uso de diferentes heteroátomos) durante esta reação é provavelmente um outro ponto de desenvolvimento desta poderosa metodologia sintética.

\section{AGRADECIMENTOS}

Aos professores, estudantes e funcionários do NPPN/UFRJ que me apoiaram, tornando minha transferência para a UFPB possível. À FAPESQ (Fundação de Apoio à Pesquisa do Estado da Paraíba) e ao CNPq (Conselho Nacional de Desenvolvimento Científico e Tecnológico) pelo financiamento.

\section{REFERÊNCIAS}

1. Class, Y. J.; DeShong, P.; Chem. Rev. 1995, 95, 1843.

2. Norcross, R. D.; Paterson, I.; Chem. Rev. 1995, 95, 2041.

3. Marko, I. E.; Bayston, D. J.; Synthesis 1996, 297.

4. Schmid, G.; Fukuyama, T.; Akasaka, K.; Kishi, Y.; J. Am. Chem. Soc. 1979, 101,259

5. Rychnovsky, S. D.; Hu, G. Y. Y.; Khire, U. D.; J. Org. Chem. 1997, 62, 3022 .

6. Nicolaou, K. C.; Theodorakis, E. A.; Rutjes, F. P. J. T.; Tiebes, J.; Sato, M.; Untersteller, E.; Xiao, X.-Y.; J. Am. Chem. Soc. 1995, 117, 1171.

7. Smith III, A. B.; Verhoest, P. R.; Kelvin, P.; Minbiole, K. P.; Schelhaas, M.; J. Am. Chem. Soc. 2001, 123, 4834.

8. Mulzer, J.; Meyer, F.; Tetrahedron Lett. 1995, 36, 3503.

9. Liu, P.; Jacobsen, E. N.; J. Am. Chem. Soc. 2001, 123, 10772.

10. Cravotto, G.; Nano, G. M.; Palmisano, G.; Tagliapetra, S.; Tetrahedron: Asymmetry 2001, 12, 707.

11. Mirtin, V. S.; Palazón, J. M.; Soler, M. A.; Ramirez, M. A.; Tetrahedron Lett. 1993, 34, 5467.

12. Edmunds, A. J. F.; Trueb, W.; Tetrahedron Lett. 1997, 38, 1009.

13. Ohkata, K.; Takagi, R.; Sasaoka, A.; Nishitani, H.; Kojima, S.; Hiraga, Y.; J. Chem. Soc., Perkin Trans. 1 1998, 925.

14. Johnson, M. R; Nakata, T.; Kishi, Y.; Tetrahedron Lett. 1979, 20, 4343.

15. Matsuda, A.; Shuto S.; Ichikawa, S.; J. Am. Chem. Soc.1999, 121, 10270.

16. Collum, D. B; McDonald, J. H.; Still, W. C. ; J. Am. Chem. Soc. 1980, 102,2117

17. Baader, E.; Bartmann, W.; Beck, G.; Bergmann, A.; Fehlhaber, H. W. Jendralla, H.; Kesseler, K.; Saric, R.; SchUssler, H.; Teetz, V.; Weber, M.; Wess, G.; Tetrahedron Lett. 1988, 29, 2563

18. Bolitt, V.; Mioskowski, C.; Bahatt, R. K.; Falk, J. R.; J. Org. Chem. 1991, 56,4238 .

19. Gruttadauria, M.; Aprile, C.; Riela, S.; Noto, R.; Tetrahedron Lett. 2001, 42, 2213.

20. Mori, Y.; Yaegashi, K.; Furukawa, H.; Tetrahedron 1997, 53, 12917.

21. Yang, W. Q.; Kitahara, T.; Tetrahedron 2000, 56, 1451.
22. Laszlo, S. E.; Ford, M. J.; Ley, S. V.; Maw, G. N.; Tetrahedron Lett. 1990, 31,5525 .

23. Arundale, E.; Mikeska, L. A.; Chem. Rev. 1952, 51, 505.

24. Adams, D. R.; Bhatnagar, S. P.; Synthesis 1977, 661.

25. Snider, B. B. Em The Prins Reaction and Carbonyl Ene Reactions; Trost, B. M.; Fleming, I., Heathcock, C. H., eds.; Pergamon Press: New York, 1991, vol. 2, p. 527.

26. Overman, L. E.; Pennington, L. D.; J. Org. Chem. 2003, 68, 7143.

27. Hart, D. J.; Bennet, C. E.; Org. Lett. 2003, 5, 1499.

28. Barry, C. S. J.; Crosby, S. R.; Harding, J. R.; Hughes, R. A.; King, C. D.; Parker, G. D.; Willis, C. L.; Org. Lett. 2003, 5, 2429.

29. Miranda, P. O.; Diaz, D. D.; Padron, J. I.; Bermejo, J.; Martin, V. S.; Org. Lett. 2003, 5, 1979.

30. Lopez, F.; Castedo, L.; Mascarenas, J. L.; J. Am. Chem. Soc. 2002, 124, 4218.

31. Crosby, S. R.; Harding, J. R.; King, C. D.; Parker, G. D.; Willis,C. L.; Org. Lett. 2002, 4, 3407.

32. Crosby, S. R.; Harding, J. R.; King, C. D.; Parker, G. D.; Willis, C. L.; Org. Lett. 2002, 4, 577.

33. Cho, Y. S.; Kim, H. Y.; Cha, J. H.; Pae, A. N.; Koh, H. Y.; Choi, J. H.; Chang, M. H.; Org. Lett. 2002, 4, 2025.

34. Yang, X.-F.; Mague, J. T.; Li, C.-J.; J. Org. Chem. 2001, 66, 739.

35. Miranda, L. S. M.; Vasconcellos, M. L. A. A.; Synthesis 2004, 1767.

36. Miranda, L. S. M.; Marinho, B. G.; Leitão, S. G.; Matheus, E. M.; Fernandes, P. D.; Vasconcellos, M. L. A. A.; Bioorg. Med. Chem. Lett. 2004, $14,1573$.

37. Miranda, L. S. M.; Meireles B. A.; Costa, J. S.; Pereira, V. L. P.; Vasconcellos, M. L. A. A.; Synlett 2005, 869

38. Prins, H. J.; Chem. Weekblad 1919, 16, 1072, 1510 (C.A. 13, 3155 (1919)).

39. Hanschke, E;. Gendorf, O.; Chem. Ber. 1955, 88, 1053.

40. Stapp, R. P.; J. Org. Chem. 1969, 34, 479.

41. Yang, X-F.; Mangue, J. T.; Li, C-J.; J. Org. Chem. 2001, 66, 739.

42. Alder, R. W.; Harvey, J. N.; Oabley, M. T.; J. Am. Chem. Soc. 2002, 124, 4960.

43. Jasti, R.; Vitale, J.; Rychnovsky, S. D.; J. Am. Chem. Soc. 2004, 126, 9904.

44. Jasti, R.; Anderson, C. D.; Rychnovsky, S. D.; J. Am. Chem. Soc. 2005, $127,9939$.

45. Crosby, S. R.; Harding, J. R.; King, C. D.; Parker, G. D.; Willis, C. L.; Org. Lett. 2002, 4, 577.

46. Crosby, S. R.; Harding, J. R.; King, C. D.; Parker, G. D.; Willis, C. L.; Org. Lett. 2002, 4, 3407

47. James, S. M.; Jaber, J. J.; Vitale, J. P.; Rychnovsky, S. D.; Org. Lett. 2002, 4, 3919.

48. De Albuquerque, I. L.; Galeffi, C.; Casinovi, C. G.; Martini-Mertolo, G. B.; Gazz. Chim. Ital. 1964, 95, 95.

49. Craveiro, A. A.; Gotlieb, O. R.; An. Acad. Brasil. Cienc. 1968, 40, 39.

50. Dobbs, A. P.; Guesné, S. J. J.; Hursthouse, M. B.; Coles, S. M.; Synllet 2003, 1740.

51. Câmara, C. A.; Pinto, A. C.; Vargas, M. D.; Zukerman-Schpector, J.; Tetrahedron 2002, 58, 6135.

52. Dobbs, A. P.; Guesné, S. J. J.; Martinovic, S; Coles, S. J.; Hursthouse, M. B.; J. Org. Chem. 2003, 68, 7880.

53. Overman, L.E.; Thompson, A. S.; J. Am. Chem. Soc. 1988, 110, 2248.

54. Kopecky, D. J.; Rychnovsky, S. D.; J. Am. Chem. Soc. 2001, 123, 8420.

55. Patterson, B.; Marumoto, S.; Rychnovsky, S. D.; Org. Lett. 2003, 5, 3163.

56. Cohen, F.; MacMillan, D. W. C.; Overman, L. E.; Romero, A.; Org. Lett. 2001, 3, 1225

57. Overman, L. E.; Pennington, L. D.; J. Org. Chem. 2003, 68, 7143.

58. Overman, L. E.; Velthuisen, E. J.; Org. Lett. 2004, 6, 3853.

59. Armstrong, A.; Shanahan, S. E.; Org. Lett. 2005, 7, 1335.

60. Costa, A. L.; Piazza, M. G.; Tagliavini, E.; Umani-Ronchi, A.; J. Am. Chem. Soc. 1993, 115, 7001 . 